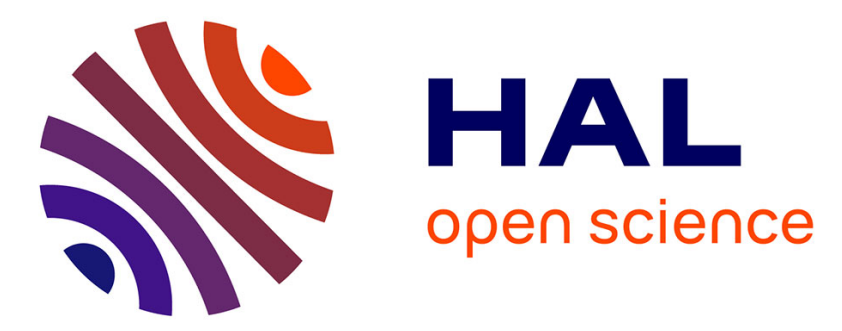

\title{
L'état de la recherche sur les mines et les métallurgies en Gaule, de l'époque gauloise au haut Moyen Âge
}

\author{
Claude Domergue, Marc Leroy
}

\section{To cite this version:}

Claude Domergue, Marc Leroy. L'état de la recherche sur les mines et les métallurgies en Gaule, de l'époque gauloise au haut Moyen Âge. Gallia - Archéologie de la France antique, 2000, Mines et métallurgies en Gaule, 57, pp.3-10. 10.3406/galia.2000.3017 . hal-01902521

\section{HAL Id: hal-01902521 \\ https://hal.science/hal-01902521}

Submitted on 20 Jan 2020

HAL is a multi-disciplinary open access archive for the deposit and dissemination of scientific research documents, whether they are published or not. The documents may come from teaching and research institutions in France or abroad, or from public or private research centers.
L'archive ouverte pluridisciplinaire HAL, est destinée au dépôt et à la diffusion de documents scientifiques de niveau recherche, publiés ou non, émanant des établissements d'enseignement et de recherche français ou étrangers, des laboratoires publics ou privés.

\section{(ㅇ)(1) $\$$}

Distributed under a Creative Commons Attribution - NonCommercial - NoDerivatives $\mid 4.0$ 


\title{
L'ÉTAT DE LA RECHERCHE SUR LES MINES ET LES MÉTALLURGIES EN GAULE, DE L'ÉPOQUE GAUlOISE AU HAUT MOYEN ÂGE
}

\author{
Claude Domergue et Marc LeRoY
}

\begin{abstract}
Mots-clés. Gaule, archéologie minière et métallurgique, sidérurgie, mines d'or, mines d'argent, mines de cuivre, archéométrie, expérimentation.

Key-words. Gaul, mining and metallurgical archaeology, ironworking, gold mines, silver mines, copper mines, archaeometallurgy, experiments.
\end{abstract}

L'archéologie minière et métallurgique n'est pas un domaine très fréquenté par les chercheurs. Elle est humble, pauvre, exigeante, difficile. Elle affectionne l'obscurité des galeries, la pénombre des forges, l'éclat noir des scories et la sombre poussière des anciennes fonderies. Elle ne découvre pas de ces «trésors » qui attirent les foules. Mais, par on ne sait quel philtre mystérieux, tous ceux qui se risquent sur ses sentiers scabreux sont marqués d'un signe auquel ils se reconnaissent. Il faut voir et entendre cette poignée d'enthousiastes dans les «sabbats » annuels auxquels les convoque l'association pour l'étude des Mines et de la Métallurgie, fondée au début des années 1980, après la réunion qui, pour la première fois, rassembla ceux qui en France commençaient alors à explorer ce domaine 1. C'était en novembre 1980, à Toulouse. L'exposé historiographique qui avait ouvert la table ronde (Pailler, 1982) rappelait

1. Cette association, créée à l'initiative des chercheurs eux-mêmes, a pour vocation de rassembler l'ensemble des archéologues et spécialistes, professionnels et amateurs, travaillant sur ce thème dans le cadre de la programmation nationale définie par le Conseil supérieur (puis national) de la recherche archéologique, placé auprès du ministre de la Culture: programme H27, puis H3 jusqu'en que, dès le XIX $\mathrm{s}$., les articles de Daubrée, encore cités aujourd'hui (Daubrée, 1868, 1881), avaient donné à cette archéologie ses lettres de noblesse et qu'après quelques décennies de léthargie, à peine troublées par quelques études marquantes (Tryon-Montalembert, 1955, 1956), les années 1970-1980 annonçaient son renouveau. La «prophétie » s'est vérifiée. En France, au cours des derniers vingt ans écoulés, l'archéologie minière et métallurgique s'est affirmée, elle s'est structurée, elle a obtenu des résultats, elle a publié, elle a organisé des colloques, elle s'est fait connaître du grand public, elle est appréciée à l'étranger. Pour s'en convaincre, il suffit de comparer le contenu des bilans de la programmation archéologique nationale, parus à intervalles réguliers entre 1981 et 1995 : l'augmentation du nombre des chercheurs ou équipes de recherche, la croissance de celui des opérations réalisées, l'évolution

1996, P25 depuis cette date, il concerne l'étude des mines et de la métallurgie (tous métaux) de la Protohistoire à l'époque contemporaine, et a depuis peu été élargi à l'ensemble de l'histoire des techniques. Nous ne considérerons dans ce dossier que les aspects touchant à la mine et à la métallurgie à l'âge du Fer, dans l'Antiquité et au haut Moyen Âge. 
quantitative et qualitative des axes de recherche, la multiplication des publications en sont les preuves indéniables ${ }^{2}$.

Encadrant cette évolution, les organismes publics de recherche - CNRS, ministère de la Culture/sousdirection de l'Archéologie, universités - se sont efforcés d'unir leurs efforts et de soutenir institutionnellement et financièrement les programmes qui leur ont été proposés. Des travaux universitaires ont pour objet des inventaires ou des monographies régionales (universités de Besançon, Compiègne, Lille, Mulhouse, Nancy, Paris I, Poitiers, Toulouse; EHESS). Mines et établissements métallurgiques antiques ont fait l'objet de fouilles programmées autorisées par le ministère de la Culture ou d'Actions thématiques programmées (ATP) financées par le CNRS. Le développement considérable des fouilles de sauvetage liées aux travaux d'aménagement du territoire a, en outre, livré une moisson de données nouvelles qu'il n'était pas facile d'obtenir par le biais des seuls moyens matériels et financiers affectés à l'archéologie programmée.

C'est dans ce contexte que des équipes se sont constituées. Elles ont une vocation diachronique et pluridisciplinaire, car cette thématique se situe au croisement de plusieurs champs de l'histoire (aussi bien économique, sociale, que technique) et fait appel à des disciplines scientifiques variées comme la géologie, la géochimie ou la métallographie, mais aussi la botanique, l'ethnologie, voire le paléoenvironnement. Dans l'est de la France, un solide pôle de recherche s'est constitué autour du Centre de recherches pétrographiques et géochimiques (CRPGCNRS) et du laboratoire d'Archéologie des métaux de Nancy, sous l'égide d'abord de l'Institut d'archéologie antique de l'université de Besançon, puis de l'UPR A0423 du CNRS, basée à l'Institut polytechnique de

\footnotetext{
2. Programmation de la recherche archéologique de terrain en France, Paris, ministère de la Culture, direction du Patrimoine/sous-direction de l'Archéologie, Conseil supérieur de la recherche archéologique, 1984, 120 p. (programme H27 : " Mines et métallurgie ", p. 77-79); La recherche archéologique en France 1985-1989, Paris, ministère de la Culture, de la Communication, des Grands Travaux et du Bicentenaire, direction du Patrimoine/sous-direction de l'Archéologie, Association pour les fouilles archéologiques nationales, 1990, $286 \mathrm{p}$. (programme H3 : « Mines et métallurgie », p. 148-153) ; La recherche archéologique en France. Bilan 1990-1994 et programmation du Conseil national de la recherche archéologique, Paris, ministère de la Culture, direction du Patrimoine/sousdirection de l'Archéologie, éd. de la Maison des sciences de l'homme, 1997,460 p. (ancicn programmc H3: "Mincs et métallurgie ", p. 174-188).
}

Sévenans; y participe aussi le groupe de travail suisse d'Archéologie du fer (GSAF). Dans le Centre, l'université de Poitiers est un autre pôle. Dans le Sud-Ouest, autour de l'UMR 5608 du CNRS, de l'université de Toulouse-Le Mirail, gravitent des laboratoires de l'université Paul-Sabatier, de l'Institut polytechnique de Toulouse et de l'École des mines d'Albi-Carmaux, des chercheurs indépendants et des établissements tels que le musée archéologique Henri-Prades (Lattes, Hérault). Ces pôles ont un rôle moteur déterminant dans la programmation et l'orientation des programmes de recherche. Ils remplissent aussi, et de plus en plus, une mission de conseil et d'expertise aux côtés des chercheurs débutants aussi bien que des équipes de terrain de l'Association pour les fouilles archéologiques nationales (AFAN), au sein de laquelle plusieurs archéologues se sont spécialisés dans ce type de recherche. Enfin, l'Association pour l'étude des Mines et de la Métallurgie intervient comme un élément fédérateur, avec ses réunions annuelles d'informations et d'échanges dans une perspective transversale et diachronique.

Les résultats n'ont pas tardé, et les publications ont suivi : en France, dans les revues régionales (Aquitania, Revue archéologique de l'Est, Revue archéologique du Centre, Revue archéologique de Narbonnaise), à l'occasion de congrès nationaux (congrès nationaux des Sociétés savantes: Grenoble 1986, Strasbourg 1988), dans des colloques spécialisés (Paris 1986 : Chevallier éd., 1987; Poitiers 1995 : Dieudonné-Glad, Nicolini éds, 1998 ; Bourg-enBresse/Beaune 1997 : Frère-Sautot dir., 1998). De nombreux chercheurs participent régulièrement aux symposiums organisés par le comité pour la Sidérurgie ancienne - Mayence, Allemagne, 1986; Liblice, Tchécoslovaquie, 1987; Ameliowka, Pologne, 1989; Sévenans-Belfort, France, 1990 ; Budalen, Norvège, 1991 ; Besançon, France, 1993 ; Norberg, Suède, 1995 ; Snowdonia Park, pays de Galles, 1997; Bienno, Italie, 1998 - ou à divers autres colloques, tables rondes et congrès réunis sur ce thème des mines et des métallurgies anciennes: à Madrid (Domergue éd., 1989a), en Yougoslavie (Jovanovic éd., 1995), en Bavière (Morteani, Northover éds, 1995), en Iran (Vatandoust éd., à paraitre), à Limoges (Cauuet, 1999), etc. Bref, entre 1980 et 1998 , les publications ont été nombreuses. On le verra dans les paragraphes suivants, où, sans prétendre à l'exhaustivité, nous voulons montrer, région par région, 
métal par métal, les principaux caractères de ces recherches (fig. 1).

Dans l'Est, autour de M. Mangin, toute une pépinière de chercheurs, partant de témoignages laissés par des praticiens du siècle dernier, ont mené des recherches sur le fer dans cette région. De nombreuses publications font connaître, souvent dans le détail et à partir d'une solide base archéométrique, une activité d'exploitation et d'extraction du fer divisée en petites unités (Mangin et al., 1992 ; Laurent, 1996 ; Leroy, 1997). Dans le sillage de P.-L. Pelet et de ses recherches sur Les Bellaires (Pelet, 1973, 1993), des travaux sur l'ancienne sidérurgie de la Suisse occidentale (Eschenlohr, Serneels, 1991 ; Serneels, 1993) renouvellent et parfois confirment les vieilles études du XIX ${ }^{\mathrm{e}} \mathrm{s}$. (Quiquerez, 1855). Un patient travail de terrain a permis de hiérarchiser les différents secteurs sidérurgiques de l'Est selon leur importance (Faivre et al., 1995 ; Mangin et al., 1995 ; Leroy et al., infra, p. 11-21). Porté à s'intéresser prioritairement au fer vu les ressources de la région, $M$. Mangin a donné sur l'exploitation du fer dans l'ancienne Gaule des synthèses documentées (Mangin, 1988, 1996) et un classement des régions productrices (Mangin, Serneels, 1996). Avec son équipe, il a publié une bibliographie rétrospective sur les mines et les métallurgics ancicnncs du fcr, qui, cn 1992, comptait 2436 titres (Paquier, Mangin, 1992). Pour sensibiliser les chercheurs aux particularités de la paléosidérurgie, V. Serneels et ses collègues du GSAF rédigent un opuscule consacré à la spécificité de cette discipline (Doswald et al., 1997).

Le fer est en effet le métal qui, en France, a le plus bénéficié des recherches récentes. Cela ne saurait surprendre l'historien, qui sait qu'en Gaule on ne connaît de procuratèles financières que pour les ferrariae (Sablayrolles, 1989a), ce qui parait être un signe de l'importance économique de ces dernières. Dans le Centre, on n'a pas encore vraiment fouillé les ferriers de la forêt d'Allogny, Cher (Landes, Bordeloup, 1985), mais on connaît mieux la production du fer chez les Bituriges (Dieudonné-Glad, 1992, 1993 ; Dumasy, 1994). Les grands travaux d'aménagement sont à l'origine de la découverte de plusieurs ensembles paléosidérurgiques, dont l'apport se révèle déterminant pour la connaissance d'aspects techniques (l'extraction des minerais de fer, la technologie des fourneaux à scorie non écoulée hors de la cuve) ou de périodes (l'âge du Fer) encore mal connus en France jusqu'à aujourd'hui :
- l'atelier des Clérimois (Yonne) qui a produit des dizaines de milliers de tonnes de scories avec des batteries de gros fourneaux entre le II ${ }^{e}$ s. avant J.-C. et le II' $s$. de notre ère, et avec des fourneaux individualisés de dimensions plus modestes aux $\mathrm{III}^{\mathrm{e}}$ et $\mathrm{IV}^{\mathrm{e}} \mathrm{s}$. (Dunikowski, Cabboï, 1995) ;

- au nord du Mans (Sarthe), de vastes secteurs d'exploitation par puits et galeries du minerai de fer cénomanien, associés à de nombreuses zones d'ateliers de réduction avec fourneaux à scories piégées ou écoulées, parmi les plus anciens actuellement découverts en France et même en Europe occidentale (du V au I ${ }^{\text {er }}$ s. avant J.-C., inédit) ; - à La Bussière (Loiret), mines à puits cuvelés et ateliers de réduction d'époque gallo-romaine (inédit) ;

- dans la région parisienne, à Vert-Saint-Denis près de Melun (Seine-et-Marne) ( $f f$. Daveau et al., infra, p. 77-99) ou en Champagne à Saint-Dizier (Haute-Marne) (Leroy, Merluzzo, 1998), des activités minières et sidérurgiques associées à des activités agropastorales au sein d'habitats de l'époque carolingienne ou féodale.

Dans le Sud-Ouest, vingt-cinq ans après les premières fouilles, le domaine des Forges (Les Martys, Aude) est devenu un site de référence ${ }^{3}$ : quelque 320 années d'activité, au moins $140000 \mathrm{~m}^{3}$ de scories et $37000 \mathrm{t}$ de fcr, des batteries de bas fourneaux et des foyers d'épuration du $\mathrm{I}^{\mathrm{er}} \mathrm{s}$. avant notre ère, des procédés techniques identifiés (le broyage des scories par exemple, of. Domergue et al., 1997). Et ce site n'est pas isolé : dans l'environnement immédiat, les ferriers abondent, ainsi que dans le secteur de Saint-Denis, Aude (Sablayrolles, 1989b) et dans toute la Montagne Noire. Dans les Pyrénées, les prospections dans le massif du Canigou (Barouillet et al., 1989) n'ont pas été suivies de fouille, sauf sur le site paléosidérurgique antique et médiéval de Saint-André-de-Baillestavy (Baillestavy, PyrénéesOrientales, $c f$. Mut, 1988) ; dans les Pyrénées centrales, aux bas fourneaux de Lercoul, III' $s$. ( $c$. Dubois, infra, p. 53-62) s'ajoutent désormais les ferriers des Baronnies (cf. Beyrie et al., infra, p. 37-52).

Si la connaissance des premières étapes de la chaîne opératoire (et davantage la réduction des minerais que l'extraction minière) a beaucoup progressé, l'étude des stades suivants (les activités de forge) n'a pas pour autant été négligée. Dans ces domaines, les découvertes sont

3. La bibliographie récente concernant ce site est donnée dans l'article de Decombeix et al., infra, p. 23-36. 


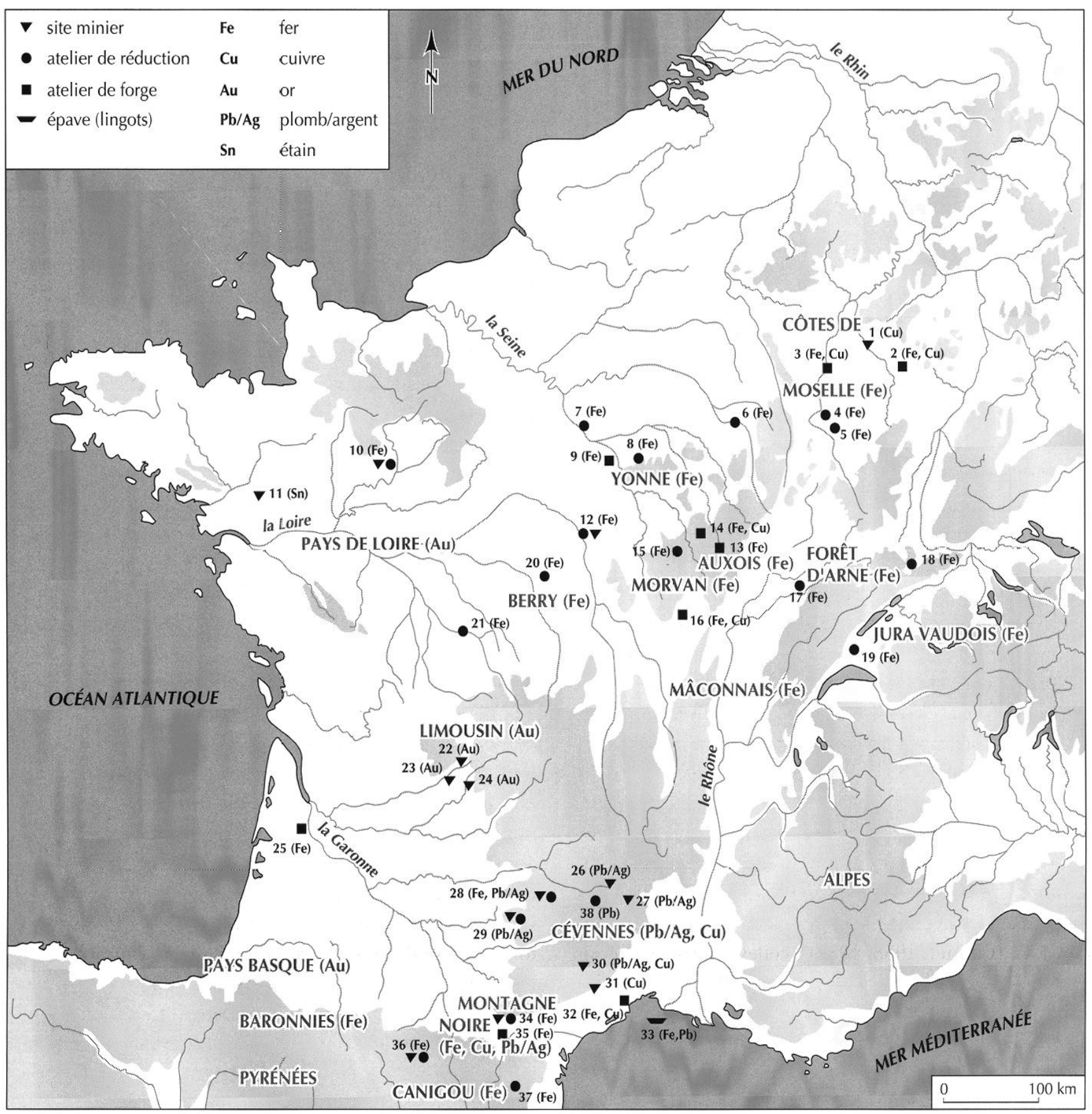

Fig. 1 - Mines et mélallurgies antiques de l'ancienne Gaule (de l'âge du Fer au haut Moyen Âge) : carte de localisation des régions productives et des principaux sites mentionnés dans le dossier.

1, Wallerfangen (Sarre, Allemagne), mine Santa Barbara (Aemiliani officina), cuivre, époque romaine ; 2, Bliesiruck (Moselle, France), agglomération d'époque romaine, ateliers de bronziers et de forgerons ; 3, Metz/Divodurum (Moselle, France), cheflieu de cité (Médiomatriques), époque romaine, ateliers de bronziers et de forgerons; 4, Frouard (Meurthe-et-Moselle, France), atelier sidérurgique, haut Moyen Âge; 5, Ludres (Meurthe-el-Moselle, France), ateliers sidérurgiques, haut Moyen Âge ; 6, Saint-Dizier (Haute-Marne, France), ateliers sidérurgiques, Moyen Âge; 7, Vert-Saint-Denis (Seine-et-Marne, France), ateliers sidérurgiques, haut Moyen Âge; 8, Les Clérimois (Yonne, France), ateliers sidérurgiques, périodes celtique et romaine; 9, Nailly (Yonne, France), atelier de forge en milieu rural, époque romaine; 10, région du Mans (Sarthe, France), mines de fer et ateliers sidérurgiques, second âge du Fer et époque romaine; 11, Abbaretz-Nozay (Loire-Atlanlique, France), mines d'étain, époque romaine; 12, La Bussière (Cher, France), mines de fer et ateliers sidérurgiques, époques romaine el médiévale; 13, Blessey (Côte-d'Or, France), agglomération d'époque romaine, ateliers de forgerons; 14, Alise-Sainte-Reine/Alésia (Côte-d'Or, France), aggloméralion d'époque préromaine et romaine, ateliers de bronziers et de forgerons; 15, Thoste (Côte-d'Or, France), atelier sidérurgique, époque romaine; 16, Autun (Saône-et-Loire, France), cheflieu de cité (Éduens), époque romaine, ateliers de bronziers el de forgerons; 17, Ferrières-les-Bois (Doubs, France), atelier sidérurgique, haul Moyen Âge ; 18, Boécourt (canton du Jura, Suisse), atelier sidérurgique, haut Moyen Âge ; 19, Les Bellaires (canton de Vaud, Suisse), ateliers sidérurgiques, fin du second âge du Fer, époque romaine et haut Moyen Âge; 20, forêt d'Allogny (Cher, France), ateliers sidérurgiques, époques romaine et médiévale; 21, Oulches (Indre, France), atelier sidérurgique, époque romaine; 22, Cros Gallet (Le Chalard, Haule-Vienne. France), mines d'or, second âge du Fer; 23, Les Fouilloux (Jumilhac-le-Crand, Dordogne, France), mines d'or, second âge du Fer; 24, La Fagassière (Château-Chervix, Haute-Vienne, France), mines d'or, second âge du Fer; 25, Bordeaux/Burdigala (Cironde, France), cheflieu de cité (Bituriges Vivisques), époque romaine, atelier de forgerons ; 26, Le Bleymard (Lozère, France), mines de plomb argentifêre, époque romaine; 27, Vialas (Lozère, France), mines de plomb argentifêre, époque romaine ; 28, Kaymar (Aveyron, France), mines de plomb argentifere 
principalement dues à l'archéologie préventive et ont surtout concerné la période antique, qu'il s'agisse de forges sịtuées dans des agglomérations (Forrières et al., 1987 ; Leroy et al., 1990 ; Lebeaupin, 1998) ou en milieu rural (Dunikowski et al., 1996; Ginouvez et al., 1998) ; celle de Blessey (Côte-d'Or), qui a fonctionné du $\mathrm{I}^{\mathrm{er}} \mathrm{au}$ IV ${ }^{\mathrm{e}}$ s., a peut-être une dimension proto-industrielle (Mangin et al., 2000b). L'atelier gallo-romain de forgerons-charrons mis au jour à Bordeaux présente de longues fosses rubéfiées, qui ont été interprétées comme des fosses de cémentation destinées à la fabrication de bandes de roulement pour roues de char (Leblanc, 1997), une découverte à laquelle répond en écho celle de structures analogues à Bram (Aude), en bordure de la voie d'Aquitaine (Passelac, 1998).

Pour les périodes antérieures à la Renaissance, les recherches sur les non-ferreux n'ont pas eu une grande ampleur jusqu'à une date récente. À l'inverse du fer, elles ont presque exclusivement concerné les techniques d'extraction des minerais (or, argent et cuivre) et n'ont que peu abordé les phases de traitement. Dans le Limousin, B. Cauuet poursuit depuis 1986 des recherches sur l'exploitation de l'or. Avant qu'elle ne les entreprît, on pouvait penser que ces aurières étaient romaines, tant on est enclin à ne prêter qu'aux riches. La surprise devait être totale. Les premières recherches en effet révèlent des mines gauloises exploitées, pour l'essentiel, du Ve au I ${ }^{\mathrm{er}} \mathrm{s}$. avant J.-C. (Cauuet, 1988, 1989) ; celles qui suivent montrent l'usage de procédés techniques avancés, dans les mines (évacuation des eaux souterraines, boisage) et dans le traitement des minerais et du métal (lavage et grillage des minerais; purification de l'or, cf. Cauuet, 1994a, 1994b). Il s'agit là d'avancées sur les techniques extractives parmi les plus importantes réalisées en Europe tempérée. Parallèlement, les anciennes mines d'or des Pyrénées (chez les Tarbelli [Strabon, 4, 2, 1], en
Ariège) et d'Auvergne commencent à être étudiées. Celles des Pays de Loire semblent devoir suivre.

Parmi les mines d'argent des Rutènes (Strabon, 4, 2, 2), celles de Villefranche-de-Rouergue ont fait l'objet de recherches (Morasz, 1989), ainsi que celles du bassin supérieur de l'Orb (Hérault). Ces dernières ont été exploitées principalement du $\mathrm{II}^{\mathrm{e}} \mathrm{s}$. avant J.-C. au $\mathrm{I}^{\mathrm{er}} \mathrm{s}$. de notre ère. Située à Lascours, au coeur du district, une petite agglomération de mineurs et de métallurgistes a été fouillée de façon discontinue. On y a en particulier découvert des tessères en plomb, qui portent le nom de la Société des mines d'argent rutènes, sans doute une compagnie fermière (Barruol, Gourdiole, 1982 ; Landes, Gourdiole, 1998). Par ailleurs, une équipe prospecte les scories plombeuses du Mont-Lozère, sous la houlette d'Alain Ploquin.

Toujours dans les contreforts méridionaux des Cévennes, les mines de Cabrières (Hérault) renfermaient les vestiges les plus anciens d'extraction du cuivre qui aient été trouvés en France : ils datent du Chalcolithique (Ambert éd., 1996). Mais ce gisement a aussi été exploité à l'époque romaine. Une nouvelle exploration, facilitée par le dénoyage temporaire des réseaux antiques, a permis des observations précises sur les conditions de travail dans des chanticrs d'ćpoquc augustéenne (Landes, Gourdiole, 1998, p. 61-63). Dans les Pyrénées, où se poursuit une recherche originale qui associe l'histoire de la forêt à celle des métallurgies anciennes (Dubois, 1994), les mines de cuivre argentifère du Séronais ont été exploitées au I ${ }^{\text {er }}$ s. avant J.-C. (Guilbaut, 1981 ; Dubois, Guilbaut, 1982, 1986). Dans les agglomérations urbaines ou les sites ruraux, ce sont surtout des ateliers de bronziers qui ont été découverts, mais bien peu ont fait l'objet d'une étude détaillée comme ceux du quartier du forum augustéen et du Haut-Empire d'Alésia (Côte-d'Or, cf. Mangin, 1981 ; Bénard, 1989) ou tout récemment du

et ateliers sidérurgiques, époque romaine, 29, Villefranche-de-Rouergue (Aveyron, France), mines de plomb argentifêre et ateliers de réduction, époques romaine el médiévale; 30, Lascours et haule vallée de l'Orb (Hérault, France), mines de cuivre et de plomb argentifêre, époque romaine; 31, Cabrières (Hérault, France), mines de cuivre, époques protohistorique et romaine; 32, Lattes (Héraul, France), agglomération d'époque préromaine el romaine, ateliers de bronziers et de forgerons; 33, Saintes-Maries-de-la-Mer (Bouches-du-Rhône, France), épaves avec lingots de fer et lingots de plomb, époque romaine; 34, Les Martys (Aude, France), mines de fer et ateliers sidérurgiques, époque romaine; 35, Bram (Aude, France), agglomération d'époque romaine, atelier de forgerons; 36, Lercoul (Ariège, France), mine de fer et atelier sidérurgique d'époque romaine; 37, Saint-André (Baillestavy, Pyrénées-Orientales, France), atelier sidérurgique, époques romaine et médiévale; 38, Le Mont-Lozère (Lozère, France), scories, Antiquité et haut Moyen Âge (?).

En aucun cas cette carte ne prétend être une cartographie exhaustive des districts miniers et métallurgiques antérieurs au bas Moyen Âge (C. Domergue et M. Leroy). 
quartier du Lycée militaire dans la ville gallo-romaine d'Autun (Chardron-Picault, Pernot, 1999).

Le bilan fait apparaître un fort déséquilibre en faveur de l'archéologie du fer. Sans doute la France est-elle riche en gisements de fer, mais les hasards de la recherche et des grands travaux, la spécialisation même des chercheurs n'y sont pas étrangers. Peut-être ces derniers ont-ils aussi bénéficié de la faveur des divinités qui protégeaient les producteurs de fer, comme Ageio dans les Baronnies ( $c f$. Beyrie et al., infra, p. 37-52) ou le dieu forgeron Gobannus en Bourgogne (Rolley, 1993). On peut en tout cas espérer que de nouveaux programmes feront bientôt sortir de l'ombre des mines aujourd'hui mal connues; on le souhaite par exemple pour l'étain du nord-Aveyron (Abraham, Morasz, 1997) ou du Limousin. Après tout, que savait-on des mines gauloises des Lemovices il y a quinze ans ? Bientôt peut-être, d'autres mines d'étain antiques s'ajouteront-elles à l'un des rares sites d'exploitation de ce métal connus en France, tel Abbaretz-Nozay (Loire-Atlantique).

Quoi qu'il en soit, le fer est par excellence le métal de la Gaule romaine. Elle en produisait des quantités, qui excédaient sans doute les consommations locales. On commence d'ailleurs à vouloir quantifier cette production pour apprécier son importancc dans lcs ćconomies antiques (cf. par exemple, Decombeix et al., 1998). Ce fer était commercialisé sous la forme de lingots; on a ainsi pensé que des lingots de fer en forme de barres allongées de la fin du $\mathrm{II}^{\mathrm{e}}$ s. avant J.-C., trouvés à Montans (Tarn), pouvaient provenir des ferrariae de la Montagne Noire (Martin, Ruffat, 1998) : c'est peut-être un peu tôt, dans l'état actuel de nos connaissances. L'exportation de métaux gaulois, en particulier du sud de la Gaule, avait été envisagée par certains (Maréchal, 1984, p. 238 et 1985, p. 246). Les lingots de fer qui constituaient les cargaisons d'épaves romaines récemment découvertes au large des Saintes-Maries-de-la-Mer (Long, 1997 ; Feugère, Serneels, 1998, p. 257-258 et 261-262) peuvent avoir une origine sud-gauloise ; cela paraît certain pour la cargaison de plomb de l'épave des Saintes-Maries-de-la-Mer 1 (Trincherini et al., à paraître).

Un trait marquant de ces recherches récentes, c'est que les travaux de terrain sont de plus en plus souvent accompagnés d'examens en laboratoire. L'étude des métaux est un des domaines où le mariage de l'archéologie et de l'archéométrie est le plus fécond. La mise sur pied, dès le milieu des années 1980, de programmes asso- ciant étroitement une démarche archéologique à une méthodologie archéométrique a placé la recherche française en paléosidérurgie à la pointe de la recherche européenne (Mangin éd., 1994, p. 9-10 ; Mangin, 1996). Le programme d'analyses PalSid, mis au point à partir de la base de données géochimique Artémise du CRPG-CNRS de Nancy, a rapidement eu une vocation nationale en centralisant la presque totalité des études de résidus de métallurgie ancienne (Ploquin, Remy, 1989 ; Ploquin, 1994b).

C'est dans l'Est qu'a été d'abord mise en chantier cette archéométrie du fer, autour des laboratoires cités plus haut. L'accent est mis sur l'étude autant descriptive qu'analytique des déchets de métallurgie (parois de fourneau, minerais plus ou moins préparés, scories coulées, scories de fond de cuve, de forge et d'affinage, battitures, déchets métalliques), afin de caractériser les procédés et de suivre leur évolution. On insiste particulièrement sur les comparaisons entre minerais et scories et sur les filiations chimiques et pétrographiques tout au long de la chaîne opératoire (Forrières et al., 1987, 1989 ; Leroy et al., 1990 ; Serneels, 1993 ; Leroy, 1997 ; Fluzin, Leclère, 1998 ; Fluzin el al., infra, p. 101-121). Le titre des Actes du colloque international de paléométallurgie de Besançon (1993) édités par M. Mangin est significatif à cet égard : La sidérurgie ancienne de l'est de la France dans son contexte européen : archéologie et archéométrie, ainsi qu'en tête de cet ouvrage, la place des articles consacrés aux techniques de laboratoire de base (Mangin éd., 1994). Le même souci anime le groupe du Sud-Ouest, avec une étroite collaboration entre archéologues et laboratoires spécialisés (Jarrier, 1993; Jarrier et al., 1995, 1996, 1997). L'archéométrie appliquée à l'étude de la mine et de la métallurgie présente une palette d'utilisation fort large, depuis les analyses de xylologie et de dendrochronologie effectuées sur les bois des mines d'or gauloises du Limousin (Cauuet, 1994b et infra, p. 129-146), jusqu'aux recettes à utiliser sur le terrain pour réveiller la mémoire chimique de certains outils métallurgiques (Morasz, Pulou, 1997) et aux techniques plus sophistiquées (le MEB) pour déterminer l'usage de gros moulins pompéiens pour broyer les scories destinées à être ajoutées à la charge des bas fourneaux du domaine des Forges (Domergue et al., 1997, p. 56-57).

La pratique de l'expérimentation, jusqu'à une date récente apanage des chercheurs anglo-saxons et centreeuropéens, est devenu très prisée en France, principalement dans le domaine des mines (Dubois, 1996 : l'abattage 
au feu) et surtout de l'archéométallurgie (DieudonnéGlad et al., 1995 ; Merluzzo, Leroy, à paraître). Les nombreuses expériences menées par P. Andrieux dans la fabrication des haches en bronze protohistoriques d'abord, dans la conduite des bas fourneaux de réduction de fer ensuite - à l'Archéodrome de Beaune, aux Martys et ailleurs (Andrieux, 1991a, 1991b, 1994; Andrieux et al., 1994) - ont rendu célèbre sa silhouette de forgeron inspiré. D'autres ont suivi cette voie, tel J.-C. Leblanc à Bordeaux, ou encore, pour le traitement des sulfures aurifères, $B$. Cauuet et $F$. Tollon. Il ne faut pas voir là un simple spectacle culturel monté par des "fans " fascinés par le spectacle du feu et de la transformation de la matière; mais retrouvcr lc savoir des Ancicns dans un domaine sur lequel ils ne nous ont laissé aucun écrit passe obligatoirement par cette étape. Chaque expérience est faite pour vérifier une hypothèse ou mesurer la conséquence d'une nouveauté technique. Ainsi en a-t-il été en septembre 1999 à Lercoul (Ariège), et en juin 1999 sur la plate-forme expérimentale du musée de la Montagne Noire à Lastours (Aude), où il s'agissait de vérifier, par diverses manipulations, l'impact qu'a sur le fonctionnement du bas fourneau « classique " des Martys l'usage de trois tuyères, attestées sur les exemplaires récemment découverts sur le site (Domergue, 1994; Domergue et al., 1999 et à paraître). Les résultats sont à l'étude.

Les avancées spectaculaires réalisées au cours de ces dernières années sur des aspects souvent neufs de cette thématique ne sauraient faire oublier que l'on a encore de nombreux progrès à faire pour comprendre la manière dont les Anciens domestiquaient ces ressources de la terre ${ }^{4}$. Mais certaines de ces recherches risquent d'être bridées, voire totalement empêchées par l'application des normes de sécurité concernant les mines anciennes ${ }^{5}$. La crainte des archéologues miniers est réelle. Elle n'a pas disparu, même après la récente convention passée entre le Bureau de Recherche géologique et minière (BRGM) et la sous-direction de l'Archéologie. Aussi, il est indispensable d'associer étroi-

4. Voir note 2, p. 4 : La recherche archéologique en France. Bilan 1990-1994 el programmation du Conseil national de la recherche archéologique, 1997, p. $404-407$.

5. Loi du 15 juillet 1994 modifiant le Code minier et rendant l'État responsable de la sécurité pour les mines orphelines. Cela conduit au dynamitage des entrées de galeries et à la destruction des installations de surface, ce qui empêche les archéologues miniers d'étudier les vestiges de réseaux miniers anciens éventuellement conservés. tement les équipes d'archéologues aux missions d'expertise du BRGM, ce qui éviterait une application systématique et aveugle du règlement, et permettrait de protéger les réseaux qu'une expertise préalable aurait jugés dignes d'intérêt (Barge, 1999).

En parcourant ce dossier, le lecteur verra qu'il ne constitue ni un ensemble cohérent, ni une synthèse complète. Il n'a pas été conçu ainsi. Il n'est l'aboutissement ni d'une recherche personnelle, ni des travaux d'une équipe. Il est la juxtaposition d'études d'importance variable, qui en sont à des stades divers de réalisation. C'est ainsi qu'il faut apprécier la différence qui existe, par exemple, entre les premiers résultats obtenus dans tel secteur minier de l'Aveyron (Kaymar) ou dans la fouille d'un petit atelier sidérurgique (Lercoul) et la synthèse des prospections qui sont menées dans l'est de la France, depuis une dizaine d'années et qui ouvrent désormais sur des perspectives d'ordre historique concernant l'organisation de la production dans cette partie des Gaules entre la fin de l'époque gauloise et le haut Moyen Âge. Mais ces deux types de travaux existent et c'est rendre compte de l'état de la recherche dans notre pays que de faire une place à l'un et à l'autre. De même, telle contribution aura besoin d'un inventaire pour asseoir son propos, alors que, pour telle autre, qui en est à un stade plus avancé, il suffira de s'y référer puisqu'il a déjà été publié. Par ailleurs, les abondantes données archéologiques fournies récemment par plusieurs chantiers de Grands Travaux ( $c f$. liste supra, p. 5) n'ont pas encore toutes fait l'objet d'études approfondies ; les chercheurs y travaillent, pas toujours avec les facilités ou les moyens adéquats. Aussi les présentations qui en sont faites, comme celle des ateliers de Vert-Saint-Denis dans ce dossier ou celle des mines et bas fourneaux de la région du Mans lors du récent colloque de l'AFEAF tenu à Nantes au printemps 1999, n'ont que la modeste ambition de livrer les premières pistes de réflexion, sans pouvoir encore proposer une synthèse plus poussée. Ainsi, ce dossier, que nous avons voulu comme une photographie aussi fidèle que possible de la réalité de la recherche, l'exprime jusque dans ses défauts...

Nous avons signalé plus haut le déséquilibre en faveur des études sur la sidérurgie. Qu'il y ait pour l'instant moins de recherches sur les mines que sur les métallurgies, que la métallurgie des non-ferreux soit moins étudiée que la sidérurgie, c'est unc rćalitć contrc laquelle nous ne pouvons pas aller et qui traduit une 
tendance actuelle, peut-être momentanée, de la recherche. Rappelons que le fer a été longtemps négligé par les archéologues. C'est maintenant le retour du balancier.

Le dernier mot sera pour remercier, au nom de tous les archéologues miniers et métallurgistes, Gallia et sa rédaction de nous avoir demandé ce dossier. Il nous paraît de bon augure que cette revue nationale de l'archéologie française, qui jusqu'ici n'avait témoigné que peu d'intérêt pour ce type de recherche (un seul article publié, nous semble-t-il, depuis sa création : Guilbaut, 1981), lui ouvre aujourd'hui largement ses pages. Bon vent à l'archéologie minière et métallurgique!

\section{Nota bene}

Pour les termes spécifiques en usage dans le domaine des mines et de la métallurgie, on se reportera au glossaire, p. 147-149. 